\section{It doesn't end here: anaesthetic recovery}

\section{Vicky Ford-Fennah}

The recovery period of anaesthesia/sedation is traditionally a period when patient monitoring and observation are stepped down following high-intensity observation during the induction and maintenance phases of anaesthesia. In 2008 the Confidential Enquiry into Perioperative Small Animal Fatalities identified that the postoperative period was associated with highest rate of fatality. Rabbits were associate with comparatively the highest risk, with $64 \%$ of all fatalities occurring in this period, followed closely by cats (61\%) and then dogs with $47 \%$. In all species the 3 -hour period after the termination of anaesthesia was the highest risk period. These findings highlight the importance of adequate patient care during the recovery period.

There are multiple reasons for why this is a high-risk period, including:

- Inappropriate timing of extubation and the appreciation of the varying requirements of different species and possible complication arising from this

- Termination of oxygen supplementation

- Residual cardiovascular and respiratory depressant effects from sedative and anaesthetic drugs

- Reduction in monitoring and use of monitoring equipment

- Hypothermia

- Time pressures on personnel

- Location of recovery

- Loss of information in the transfer of care between personnel.

Although there is a potential risk for all patients during recovery some patient groups are at particularly high risk. These include:

- Brachycephalic patients (all species) - these patients are at a higher risk of airway obstruction

- Hypothermic patients

- Patient with concurrent disease which may result in a prolonged action of drugs, for example, hepatic disease

- Patients that have undergone head or neck surgery, particularly those which have either excessive oral secretion or haemorrhage, for example, dental extraction, neck bandages or swelling resulting in restriction of the soft tissues of the upper airways or reduced airway accessibility, for example, jaw fractures and management

- Patients that have regurgitated or are at high risk of regurgitation, for example, gastric dilatationvolvulus
- Patients that have had issues maintaining oxygen saturation during anaesthesia or suffering from anaemia

- Patients that have received a neuromuscular

blocking agent

- High ASA rating

- Intraoperative haemorrhage or anaesthetic/surgical complications.

In these high-risk patients, location of recovery is paramount. Ensure you have all equipment (e.g. monitoring equipment, re-induction and intubation kits, oxygen supplementation, suction) along with trained personnel to identify problems at the earliest opportunity and to monitor these patients constantly. This process needs to occur in a location which enables help to be sought immediately if required. Often the best location for this is either the theatre or induction/prep area if an intensive care unit area/kennel is not available.

Although complications are more likely in these high-risk patients, the recovery period should be considered a high-risk period for all patients. The importance of adequate monitoring for all patients must not be underestimated.

\section{KEY LEARNING OBJECTIVES}

- Understand that recovery from anaesthesia is a comparatively high risk period and why this is

- Have an understanding of which patients are particularly high risk during recovery

- Have an understanding of techniques that can be used to maximize patient care during the recovery period and minimize the risks

\section{MULTIPLE CHOICE QUESTIONS}

1. When should a cat be extubated?
(A) On the return of the gag reflex
(B) On head lift
(C) Before the return of the gag reflex
(D) Return of pedal withdrawal reflex

2. When should the cuff on a dog's endotracheal tube be deflated?
(A) Once the administration of volatile agent ceases
(B) When the patient is disconnected from oxygen
(C) On the return of the palpebral reflex
(D) On return of the gag reflex

3. What is the most useful piece of monitoring equipment in the early stages of recovery?
(A) Capnograph
(B) Pulse oximeter
(C) Doppler
(D) Electrocardiogram 